\title{
EXPERIENCIAS MUSICALES A TRAVÉS DEL RELATO AUTOBIOGRÁFICO EN LA FORMACIÓN DOCENTE
}

\author{
Baikune De Alba-Eguiluz ${ }^{\mathrm{I}}$ \\ D Cristina Arriaga-Sanz ${ }^{\text {II }}$ \\ María Elena Riaño-Galán III \\ I Universidad del País Vasco/Euskal Herriko Unibertsitatea, Bilbao, España; baikune.dealba@ehu.eus \\ II Universidad del País Vasco/Euskal Herrriko Unibertsitatea, Bilbao, España; cristina.arriaga@ehu.eus \\ III Universidad de Cantabria, Santander, Cantabria, España; elena.riano@unican.es
}

\section{Resumen}

La música ha demostrado ser un valioso instrumento para el autoconocimiento y el desarrollo emocional de la persona. Este estudio exploratorio-descriptivo indaga sobre las experiencias musicales vitales de un grupo de estudiantes del Grado de Maestro en Educación Primaria de la Universidad de Cantabria (España) y describe los significados y vínculos otorgados a la música en relación con su futura labor docente, así como posibles líneas de actuación pedagógica. Mediante la narración de relatos autobiográficos y la reflexión en grupos de discusión, los resultados muestran la relevancia de la música en las experiencias previas e identificación de emociones y sentimientos, proporcionan información sobre contextos y estilos y ofrecen una concepción de la música como recurso educativo con un fuerte potencial emocional y creativo.

FORMACIÓN DE PROFESORES • EDUCACIÓN MUSICAL・NARRATIVAS PERSONALES

\section{EXPERIÊNCIAS MUSICAIS ATRAVÉS DE NARRAÇÕES AUTOBIOGRÁFICAS NA FORMAÇÃO DE PROFESSORES}

\section{Resumo}

A música tem se mostrado um instrumento valioso para o autoconhecimento e desenvolvimento emocional. Este estudo exploratório-descritivo investiga as experiências de vida musical de um grupo de estudantes de Formação de Professores do Ensino Fundamental [Grado de Maestro en Educación Primaria] da Universidad de Cantabria (Espanha), descrevendo os significados e vínculos dados à música em relação ao seu futuro trabalho docente, bem como as possíveis linhas de ação pedagógica. Através da narração de histórias autobiográficas e da reflexão em grupos de discussão, os resultados mostram a relevância da música em experiências anteriores e a identificação de emoções e sentimentos, fornecem informações sobre contextos e estilos, e oferecem uma concepção da música como um recurso educativo com forte potencial emocional e criativo. 


\section{MUSICAL EXPERIENCES THROUGH AUTOBIOGRAPHICAL NARRATIVES IN INITIAL TEACHER TRAINING}

\section{Abstract}

Music has proven to be a valuable tool for deepening self-knowledge and emotional development. This exploratory-descriptive study investigates the musical life experiences of a group of students on the Primary Education Teacher's Degree at the University of Cantabria (Spain), describing the meanings they ascribe to musical experience and the links they see between music and their future teaching work, as well as possible lines of pedagogical action. Through an analysis of the autobiographical narratives and reflective discussion groups, the results show the relevance of music in prior experiences and in the identification of emotions and feelings, provide information on contexts and styles, and offer a perspective of music as an educational resource with strong emotional and creative potential.

TEACHER EDUCATION • MUSIC EDUCATION • PERSONAL NARRATIVES

\section{RECITS AUTOBIOGRAPHIQUES D’EXPÉRIENCES MUSICALES DANS LA FORMATION DES ENSEIGNANTS}

\section{Résumé}

La musique s'est avérée être un instrument précieux pour la connaissance de soi et le développement émotionnel. Cette étude exploratoire-descriptive porte sur les expériences de vie musicale d'un groupe d'étudiants du Grado de Maestro en Educación Primaria de la Universidad de Cantabria (Espagne), en décrivant les significations et les liens attribués à la musique en rapport avec leur futur travail d'enseignement, ainsi que les lignes d'action pédagogique possibles. Par le biais de récits autobiographiques et de la réflexion en groupes de discussion, les résultats montrent la pertinence de la musique dans les expériences précédentes et l'identification des émotions et des sentiments, fournissent des informations sur les contextes et les styles et proposent une conception de la musique comme ressource éducative à fort potentiel émotionnel et créatif.

FORMATION DES ENSEIGNANTS • ÉDUCATION MUSICALE • RECITS AUTOBIOGRAPHIQUES 
ESE A QUE LA MÚSICA ESTÁ PRESENTE EN LOS PLANES DE ESTUdIO DE LA FORMACIÓN inicial de maestros (García \& Lorente, 2017) no siempre es abordada teniendo en cuenta su dimensión emocional (Campayo \& Cabedo, 2016) y sus posibilidades para profundizar en el autoconocimiento de la persona (Colomo \& Domínguez, 2015). En los últimos años ha sido creciente la investigación en relación con el uso de los incidentes críticos en la educación superior (Sánchez-Sánchez \& Jara-Amigo, 2015). Algunos estudios centran su foco en las experiencias subjetivas de alto impacto emocional capaces de transformar a la persona, que inciden en el modo en que se comprende y se reconoce a sí misma y la sitúan ante nuevas posibilidades de significación y posicionamiento (Valdés et al., 2016).

Este tipo de experiencias fomenta la reflexión, la observación y la identificación de elementos y eventos que pueden influir y favorecer su aprendizaje y que Burnard (2005) define como "encuentros musicales significativos". Se trata de experiencias musicales previas que han tenido una especial incidencia en la vida de cada estudiante y cuyo objetivo es visualizar el itinerario musical personal, reflexionando sobre los episodios que han marcado cada trayectoria vital particular.

Este estudio explora los significados de la música y las experiencias vitales de un grupo de estudiantes del Grado de Educación Primaria ${ }^{1}$ que se imparte en la Universidad de Cantabria (España) por medio de dos objetivos: 1) indagar acerca de las experiencias musicales de los futuros maestros mediante un proceso autorreflexivo; 2) describir cuáles son los significados y los vínculos que estos estudiantes otorgan a la música en relación con su futura labor docente.

\section{Revisión de literatura}

\section{La música como experiencia transformadora}

La descripción de experiencias previas, la evocación de recuerdos y de actividades realizadas en contextos que resultan particularmente significativos, conectan a las personas consigo mismas y contribuyen al desarrollo personal. Las experiencias transformadoras se han abordado desde distintos enfoques (Valdés et al., 2016), todos ellos centrados en el autoconocimiento y el desarrollo personal a partir de experiencias subjetivas. La reflexión sobre estas vivencias se configura como un factor fundamental para un aprendizaje valioso y significativo que parte de la experiencia personal.

En este sentido, los incidentes críticos se muestran como uno de los planteamientos con mayor vinculación con la educación, ya que explora el efecto de experiencias transformadoras y su potencialidad como agentes de mejora del proceso de enseñanza-aprendizaje. Un incidente crítico es un suceso inesperado, positivo o negativo, emocionalmente impactante en la vida de una persona. Su uso en el ámbito educativo favorece el aprendizaje transformacional y la reflexión crítica (Monereo et al., 2015). Los incidentes críticos nos interesan especialmente porque pueden llevar a un cambio en la identidad profesional del futuro docente y de los elementos que la conforman, ya sean relacionados con la instrucción como con las estrategias e inclusive las interpretaciones sentimentales que acompañan a estas estrategias (Sockman \& Sharma, 2008; Monereo \& Badía, 2011). Además, "puede resultar

1 La formación de docentes de enseñanza en las etapas de Infantil (hasta los 6 años) y Primaria (hasta los 12 años) se imparte en un Grado universitario de 4 años de duración y un mínimo de 240 créditos ECTs, ofrecido por las universidades españolas con las denominaciones de Grado en Maestro/Grado en Magisterio/Grado en Educación Infantil o en Educación Primaria. http://www. educacionyfp.gob.es/contenidos/estudiantes/portada.html; https://www.educacion.gob.es/notasdecorte/busquedaSimple.action 
de utilidad como instrumento de intervención para provocar la toma de conciencia de las personas implicadas en el incidente crítico, valorando las actuaciones desencadenadas, y para proponer, y en su caso instaurar, actuaciones alternativas frente a sucesos similares" (Bilbao \& Monereo, 2011, p. 138).

Sin embargo, la significación de un incidente no se halla en el evento en sí, sino en la interpretación que la persona hace de él. Esto da lugar a que los incidentes críticos se conviertan en un instrumento para la reflexión personal, facilitando los procesos de aprendizaje (Tripp, 2012). Una reflexión que posibilitará la comprensión de la propia identidad y constituirá la base sobre la que fundamentar la construcción del futuro rol docente (Riaño, 2017).

La fuerte conexión existente entre la música y las emociones, y el destacado papel que estas desempeñan a la hora de rememorar, describir e integrar en nuestro bagaje personal las experiencias vividas (Hebert, 2009), son aspectos a tener en cuenta no sólo en la educación musical, sino en la educación en general. Un planteamiento donde la música sirve a las personas para "usar y construir experiencias valiosas para su propia vida y formación integral, desde la experiencia artística musical" (Touriñán \& Longueira, 2010, p. 157).

Más aún, algunos estudios subrayan la necesidad de plantear la educación musical como un espacio para fomentar el desarrollo de la capacidad de reflexión, responsabilidad, trabajo o crítica, imprescindibles en la formación de una ciudadanía activa en la sociedad (Regelski \& Gates, 2009) y que le permitan responder con confianza a los distintos modos de actuación que le exige el contexto social en que se encuentra (Fernández-Jiménez \& Jorquera-Jaramillo, 2017).

Algunos estudios inciden en los beneficios que supone para el aprendizaje y la capacidad de reflexión de los estudiantes dejar que afloren sus voces y que expresen sus experiencias sobre la música, el aprendizaje musical y su vida escolar (Burnard \& Björk, 2010). El descubrimiento de acontecimientos musicales personales y la búsqueda de conexiones indujeron a la reflexión sobre los significados de las experiencias vividas anteriormente. Una forma de entender la construcción de la propia identidad (Burnard, 2005; Adler, 2012), de llegar a un autoconocimiento basado en la experiencia y altamente contextualizado.

\section{Narrativas personales}

La elaboración de una narración personal proporciona una estructura para nuestro sentido del yo y de la identidad, ya que al contar relatos sobre nuestras experiencias personales se crea también una identidad narrativa (Sparkes \& Devís, 2007) y se provocan procesos de reflexión sobre por qué uno piensa, siente y actúa de una manera determinada (Bolívar et al., 2001; Silvennoinen, 2001). Los relatos sobre cómo nos sentimos, o sobre nuestra opinión acerca de planteamientos e ideas, nos definen frente a otros, por lo que revisten gran importancia en la construcción identitaria de la persona (Baker, 2005, 2014).

Cuando se nos ofrece la oportunidad de explorar la presencia de la música en determinados momentos de nuestras vidas, somos capaces de reconectar con esas experiencias, en un proceso que también puede ser escuchado y valorado por los otros, a la vez que surge la empatía mientras se intercambian e interactúan las respectivas narraciones (Richardson, 2012). La narración autobiográfica se convierte en un elemento más en el proceso del autoconocimiento, de la creación de la identidad personal y profesional (Carrillo \& Vilar, 2016). Se trata de situar y valorar la música, presente de una forma o de otra en la vida de las personas, sean estudiantes, futuros docentes (Adler, 2012) o músicos (Pitts, 2012). Una formación, que da cabida a experiencias y subjetividades, permite que las identidades se vayan cimentando, se reconstituyan y se transformen (Castañeda, 2013; Philpott, 2016).

Por otra parte, las narraciones individuales tienen también un carácter social ya que conectan las identidades personales con el entorno sociocultural en que se desarrollan, en un enfoque tridimensional que comprende la visión temporal, la interacción persona-sociedad y el contexto espacial donde se desarrollan (Garvis \& Pendergast, 2012). Compartir las propias vivencias con otras personas que se 
encuentran en una misma situación -en este caso que comparten el deseo de ser maestros- constituye una práctica comunicativa, reflexiva y dialógica que enriquece todo el proceso (Bolívar \& Domingo, 2006; Beineke, 2012; Castañeda, 2013). Es una mirada democrática, donde la participación y la voz del alumnado es considerada como una herramienta en la construcción de una nueva cultura profesional docente (Ceballos \& Susinos, 2014).

\section{Método}

\section{Diseño}

Se siguió un paradigma cualitativo (Denzin \& Lincoln, 2012) aplicado al ámbito artístico (Luque \& Cruz, 2016) y se adoptó un diseño exploratorio-descriptivo mediante un enfoque autobiográfico autorreflexivo (Cornejo et al., 2008; Verd \& Lozares, 2016).

\section{Instrumentos}

Para recabar los datos se utilizaron dos instrumentos: el relato autobiográfico, relacionado con el primer objetivo, nos ha permitido indagar acerca de las experiencias musicales de los futuros maestros. El segundo instrumento fue el grupo de discusión, vinculado con el segundo objetivo, sobre las opiniones de los participantes acerca de los significados que otorgan a la música.

\section{Participantes}

Participó un grupo de 34 estudiantes (28 mujeres y 6 hombres) del primer curso del Grado de Educación Primaria de la Universidad de Cantabria (España), de edades comprendidas entre 18 y 20 años.

\section{Procedimiento}

El trabajo exploratorio se desarrolló a lo largo de ocho semanas, durante el horario de clase. El estudio constó de dos fases (ver Figura 1). En la primera, se solicitó a los estudiantes que escribieran relatos escritos donde narrasen experiencias significativas en las que la música hubiera desempeñado un papel destacado. Se les sugirió que describieran el máximo detalle del incidente crítico. La información incluida en las producciones elaboradas por el alumnado se sometió a un análisis inductivo de contenido (Lorenzo, 2011; Andréu, 2001) y posterior construcción de un discurso interpretativo. Se priorizaron los elementos de los discursos que permitieran extraer una serie de ideas comunes o tópicos recurrentes en las narraciones de los participantes a partir de un proceso de codificación temática (Angrosino, 2012). Un mismo tópico podría estar formado por grupos de frases con elementos en común. Las categorías englobaron a los tópicos, conformándose como grandes agrupaciones conceptuales.

En la segunda fase, los resultados obtenidos en cada categoría se presentaron como detonadores, en forma de preguntas, para su debate en grupos de discusión a lo largo de 60 minutos. Se formaron tres grupos, mixtos, dos de ellos de doce personas y un tercero integrado por diez participantes. Los temas principales del debate giraron en torno a: 1) los significados de la música en las experiencias significativas previas de los estudiantes, 2) su vinculación con su futuro ejercicio profesional, 3) diferentes líneas de actuación pedagógica.

Siguiendo a Callejo (2001), se respetaron las especificidades que caracterizan a un grupo de discusión, situándolo en un contexto en el que los discursos de los participantes pudieran fluir con libertad para facilitar la interacción personal entre sus integrantes. El espacio físico se configuró de manera que el contacto visual se viera favorecido para promover su participación en la conversación. Como paso previo al tratamiento de la información, cabe reseñar los métodos de registro y la transcripción llevados a cabo. Con respecto al primero se hicieron grabaciones de audio excluyendo el registro visual por dar 
un mayor anonimato a las fuentes de información. Todo ello fue transcrito a partir de las denominadas transcripciones artesanales que proponen Farías y Montero (2005), por constituir un primer paso para familiarizarnos con los datos recabados y tomar conciencia de la complejidad existente en el paso del lenguaje oral al lenguaje escrito. La información obtenida se analizó siguiendo un proceso similar al llevado a cabo en la primera fase para el análisis textual.

FIGURA 1

FASES DEL ESTUDIO

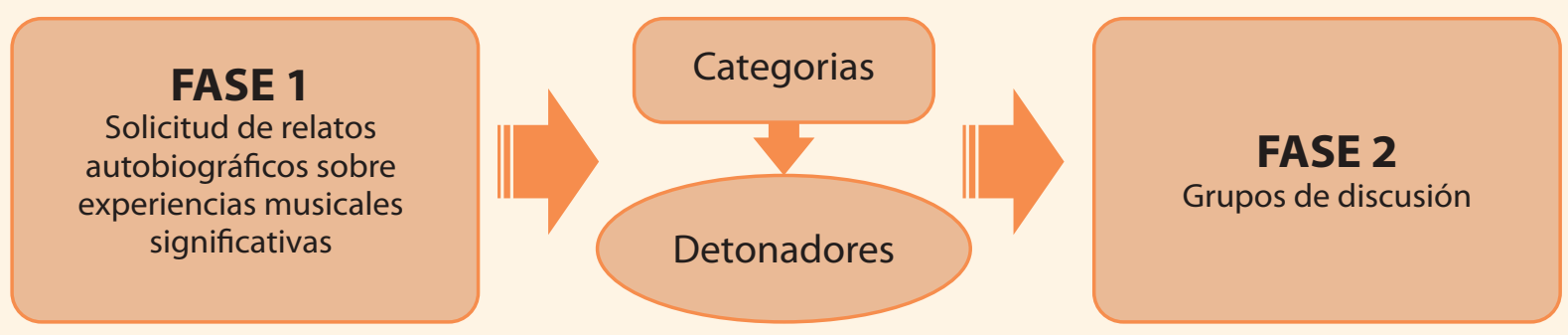

Fuente: Elaboración propia.

\section{Resultados}

\section{Resultados del análisis de los relatos}

Al relatar sus experiencias el alumnado asoció la presencia de la música mayoritariamente con estados de ánimo de relajación (13,2\%), diversión y evasión (10,5\%) y disfrute (7,9\%) y con menor frecuencia, seguridad, euforia, sorpresa y bienestar. Asociadas a estos, emociones como alegría, tristeza, energía y felicidad, entusiasmo y evocación están presentes en los relatos (5,3\%),

Los participantes relacionaron la música con prácticas como la escucha (30\%), el baile $(18,3 \%)$, el canto e interpretación instrumental (16,7\%), el desarrollo rítmico y la creación (5\%), así como la expresión corporal, la exploración o la imaginación.

El alumnado identificó una variedad de estilos al señalar la presencia de la música en sus experiencias significativas, entre los que se encuentran con mayor presencia la clásica $(11,5 \%)$, seguida de rock, pop, tradicional, musicales y bandas sonoras (7,7\%). También se nombraron los estilos electrolatino, reggaetón, bachata, rap y trap.

Otros tópicos surgidos, tras el análisis textual de los relatos, se articularon alrededor de los contextos donde el alumnado situó esas experiencias significativas: educativo formal (38,7\%), la familia $(16,1 \%)$, amistades, entorno social y medios de comunicación (el 12,9\% cada uno) y contexto educativo no formal $(6,5 \%)$. Se señaló que, en el contexto formal escolar, la música era beneficiosa como parte del desarrollo profesional y enriquecimiento cultural personal $(29,4 \%)$ y, sobre todo, como recurso para trabajar en el aula $(70,6 \%)$ mediante la educación en valores, las relaciones interpersonales, el trabajo en equipo, de manera interdisciplinaria o, inclusive, como recurso mnemotécnico.

El 54,8\% de las experiencias narradas se situaban en la franja de edad comprendida entre los 6 y 12 años, coincidente con la etapa de primaria. La franja de 13-17 años aparecía en el 32,3\% de los relatos autobiográficos y, en menor medida, ubicaron sus experiencias en edades comprendidas entre 3-5 años (9,7\%) y más de 18 años de edad (3,2\%). 


\begin{tabular}{|c|c|c|c|c|c|}
\hline Categorías & $\begin{array}{l}\text { N. }{ }^{\circ} \text { referencias } \\
\text { a categorías }\end{array}$ & $\%$ & Tópicos & $\begin{array}{l}\text { Subtotal } n .^{\circ} \\
\text { referencias a } \\
\text { tópicos }\end{array}$ & $\begin{array}{l}\text { Subtotal \% } \\
\text { tópicos }\end{array}$ \\
\hline \multirow{20}{*}{$\begin{array}{l}\text { Emociones y estados } \\
\text { de ánimo }\end{array}$} & \multirow{20}{*}{38} & \multirow{20}{*}{18,8} & Relajación & 5 & 13,2 \\
\hline & & & Evocación & 2 & 5,3 \\
\hline & & & Diversión & 4 & 10,5 \\
\hline & & & Evasión & 4 & 10,5 \\
\hline & & & Seguridad & 1 & 2,6 \\
\hline & & & Felicidad & 2 & 5,3 \\
\hline & & & Euforia & 1 & 2,6 \\
\hline & & & Entusiasmo & 1 & 2,6 \\
\hline & & & Sorpresa & 1 & 2,6 \\
\hline & & & Bienestar & 1 & 2,6 \\
\hline & & & Descanso & 1 & 2,6 \\
\hline & & & Alegría & 2 & 5,3 \\
\hline & & & Energía & 2 & 5,3 \\
\hline & & & Descarga de adrenalina & 1 & 2,6 \\
\hline & & & Tristeza & 2 & 5,3 \\
\hline & & & Tranquilidad & 1 & 2,6 \\
\hline & & & Disfrute & 3 & 7,9 \\
\hline & & & Gusto & 2 & 5,3 \\
\hline & & & Desinhibición & 1 & 2,6 \\
\hline & & & Consuelo & 1 & 2,6 \\
\hline \multirow{11}{*}{ Prácticas musicales } & \multirow{11}{*}{60} & \multirow{11}{*}{29,7} & Escucha & 18 & 30 \\
\hline & & & Interpretación instrumental & 10 & 16,7 \\
\hline & & & Canto & 10 & 16,7 \\
\hline & & & Baile & 11 & 18,3 \\
\hline & & & Creación & 3 & 5 \\
\hline & & & Coordinación & 1 & 1,7 \\
\hline & & & Desarrollo rítmico & 3 & 5 \\
\hline & & & Expresión corporal & 1 & 1,7 \\
\hline & & & Exploración & 1 & 1,7 \\
\hline & & & Coreografía & 1 & 1,7 \\
\hline & & & Imaginación & 1 & 1,7 \\
\hline \multirow{17}{*}{ Estilos } & \multirow{17}{*}{25} & \multirow{17}{*}{12,3} & Rock & 2 & 7,7 \\
\hline & & & Bandas sonoras & 2 & 7,7 \\
\hline & & & Pop & 2 & 7,7 \\
\hline & & & Reggaetón & 2 & 7,7 \\
\hline & & & Musicales & 2 & 7,7 \\
\hline & & & Jazz & 1 & 3,8 \\
\hline & & & Tradicional & 2 & 7,7 \\
\hline & & & Electro-latino & 1 & 3,8 \\
\hline & & & Bachata & 1 & 3,8 \\
\hline & & & Rap & 1 & 3,8 \\
\hline & & & Trap & 1 & 3,8 \\
\hline & & & Hip-hop & 1 & 3,8 \\
\hline & & & Punk & 1 & 3,8 \\
\hline & & & Clásica & 3 & 11,5 \\
\hline & & & Cantautores & 1 & 3,8 \\
\hline & & & Baladas & 1 & 3,8 \\
\hline & & & Otros & 1 & 3,8 \\
\hline
\end{tabular}




\begin{tabular}{|c|c|c|c|c|c|}
\hline Categorías & $\begin{array}{l}\text { N. }{ }^{\circ} \text { referencias } \\
\text { a categorías }\end{array}$ & $\%$ & Tópicos & $\begin{array}{l}\text { Subtotal n. } \\
\text { referencias a } \\
\text { tópicos }\end{array}$ & $\begin{array}{l}\text { Subtotal \% } \\
\text { tópicos }\end{array}$ \\
\hline \multirow{6}{*}{ Contextos } & \multirow{6}{*}{31} & \multirow{6}{*}{15,4} & Familia & 5 & 16,1 \\
\hline & & & Amigos & 4 & 12,9 \\
\hline & & & Educación formal & 12 & 38,7 \\
\hline & & & Medios & 4 & 12,9 \\
\hline & & & Entorno social & 4 & 12,9 \\
\hline & & & Educación no formal & 2 & 6,5 \\
\hline \multirow{2}{*}{$\begin{array}{l}\text { Beneficios ámbito } \\
\text { educativo }\end{array}$} & \multirow{2}{*}{17} & \multirow{2}{*}{8,4} & Desarrollo personal y profesional & 5 & 29,4 \\
\hline & & & Recurso en el aula & 12 & 70,6 \\
\hline \multirow{4}{*}{ Edad } & \multirow{4}{*}{31} & \multirow{4}{*}{15,4} & 3-5 años & 3 & 9,7 \\
\hline & & & 6-12 años & 17 & 54,8 \\
\hline & & & 13-17 años & 10 & 32,3 \\
\hline & & & $>18$ años & 1 & 3,2 \\
\hline TOTAL & 202 & 100 & & & \\
\hline
\end{tabular}

Fuente: Elaboración propia.

\section{Resultados del análisis de los grupos de discusión}

El objetivo 2 era describir los significados y vínculos que los participantes otorgan a la música como futuros docentes. El siguiente relato recoge los principales puntos debatidos entre los tres grupos:

La mayoría de estudiantes incidió en que la música propiciaba la introspección personal. Por ejemplo, Marta señaló: "A través de la música se consigue una conexión con uno mismo, te conecta con tu interior" y Paula expresó: "Con la música te evades, te relajas al llegar a casa. Te tranquilizas del estrés del día. Esto es más positivo que negativo”. Además de la música, el silencio fue también valorado como elemento de indagación para Lydia: "El silencio es interesante, para relajarte, para meditar..., es como un tiempo para el autoconocimiento".

Hubo acuerdo general en la identificación entre música y emociones. Ana manifestó: "Es lo que busco cuando escucho música con ritmo, la descarga de adrenalina para animarme y cuando estoy triste, pongo una música más lenta". En opinión de Juan: "Yo he sentido todas las emociones en función de las características de la música”. Y Pedro y Álvaro afirmaron que la música servía "para identificar y gestionar las emociones, conocer cómo funcionas, por qué te apetece escuchar una música determinada”, "el vehículo para ponernos de acuerdo en lo que sentimos es el diálogo, para conocer lo que sentimos cada uno en cada momento, y reconocer la diversidad de emociones ante un mismo estilo musical".

Los participantes pusieron en valor la música como recurso para el aula. En su opinión, la educación emocional es un aspecto fundamental y la música puede ser "un vehículo, no el único, pero sí imprescindible” (Jesús). En la misma línea, "a través de la música el profesor puede conocer mejor el perfil del alumno" (Carla).

Se reconoció ampliamente que este tipo de trabajo ha de hacerse desde la infancia y los futuros docentes valoraron la etapa de Primaria como la más idónea pues, "es un momento importante en la construcción de la persona y donde el profesorado es una gran referencia para niños y niñas" (Rebeca). Todos los grupos convinieron en la importancia de fomentar actividades musicales en esta etapa. María creía que "la etapa de Primaria es una edad muy bonita para descubrirse uno mismo, la música nos puede ayudar a enseñar a los niños a ser creativos y a expresar, a través de la música, cualquier sensación que ellos tienen". De la misma opinión fue Inés, quien argumentó que "es fundamental fomentar la música en Primaria. No hay tanto que enseñar como que descubrir la música a los niños y que vayan explorando" destacando así la labor introspectiva al igual que Jesús, al señalar que "es fundamental que los niños investiguen para que puedan sacar lo que tienen dentro". 
Los recuerdos que varios participantes tenían sobre las clases de música recibidas en su etapa escolar, sin embargo, revelaban la ausencia de enfoques prácticos. Algunas afirmaciones fueron: "teníamos el aula llena de instrumentos pero nunca los llegamos a tocar" (Celia), "las clases eran muy repetitivas y dábamos más teoría que tocar los instrumentos" (Sole), "antes era todo más teórico. No hacíamos ejercicios ni bailábamos" (Nuria); e, inclusive, hubo una opinión que equiparó el enfoque teórico de un docente de música con una ausencia de vocación: "Los de música parece que odiaban dar clase, dábamos más teoría que tocar los instrumentos” (Ana).

Por el contrario, las experiencias de mayor disfrute con la música en las que incidieron ciertos estudiantes estaban vinculadas a los eventos y celebraciones escolares especiales. Juan informó que "en Primaria, las actuaciones eran algo creativo, nos motivaba mucho, nos acercaba a la música". Sonia recalcó que "las experiencias musicales no han sido en la clase de música, sino en actividades extraescolares de música en el colegio" y, en la misma línea, Aitor apuntó que "eran las fiestas que preparábamos en Navidad y Semana Santa cuando todos tocábamos los instrumentos".

Todos los participantes estuvieron de acuerdo en que la edad era un factor importante que determinaba el tipo de experiencias musicales, y la adolescencia emergió como una etapa donde la influencia de los amigos o la posibilidad de disponer de un teléfono propio o salir, determinaba prácticas musicales como la de escuchar. Algunas afirmaciones al respecto fueron: "de pequeño no tienes tu teléfono, a partir de los 12 años ya puedes escuchar lo que quieres" (Javier) "porque escuchas lo que escuchan tus amigos" (Aitor) "escuchas cuando sales de fiesta" (Elsa).

Se valoró el contex to familiar como el ámbito donde los participantes se iniciaron en la escucha, se aficionaron a la música y fueron construyendo sus gustos. Rosa comentó: "mi hermano escuchaba lo mismo que yo, pero a partir de los 8 años, ya iba buscando lo que a él le interesaba”. Lucía señaló que en las reuniones familiares "escuchas la música que escuchan los primos, pero luego vas construyendo tus gustos”. Otros estudiantes dijeron tener como referentes las aficiones musicales de sus padres. Así lo expresó Carmen: "Yo siempre escuchaba la música de la época de mis padres. Ahora voy a bares y escucho otra música".

Como futuros docentes, los participantes debatieron ampliamente sobre el potencial de la música en la educación ofreciendo algunas propuestas que incluían tener en cuenta la diversidad que, hoy en día, existe en las aulas y la participación del alumnado. Luisa señaló, al respecto, "el alumnado puede proponer la música que quiere traer al aula, como un modo de conocer otros estilos musicales y para acercarse a otras culturas: "Es bueno porque a veces no te planteas escuchar cosas si no te las proponen. Cuanto más sepas, mejor te conoces a ti mismo, te da más oportunidades". Para Maite: "Ahora que hay tanta diversidad de culturas, de otros países, está bien que traigan lo que escuchan en casa, para que los demás oigan algo que normalmente no escuchan, de África, o música tradicional de aquî" y según Gema: "Que ellos traigan músicas que escuchan en familia puede aportar, porque habrá algunos que vengan de otros países... es interesante, te ayuda a conocer el entorno”.

Algunos estudiantes se interesaron por utilizar la música en colaboración con el resto del profesorado (generalistas y especialistas de diferentes asignaturas): "la cosa es juntarse, hablar y empezar a usar la imaginación para trabajar juntos, coordinarse entre los profesores para explorar todas las posibilidades" (Berta). Potenciar la interdisciplinariedad fue un aspecto en el que coincidieron varios participantes, señalando que todos los contenidos y todas las asignaturas se pueden trabajar mediante la música (Matemáticas, Lengua, Ciencias, Tecnología...), para trabajar las emociones y temas transversales como la educación en valores. O también utilizándose como recurso mnemotécnico. María se expresó: "Se puede usar la música para aprender otros contenidos, como una canción, por ejemplo. Así aprendes sin darte cuenta. Además, si esa canción te gusta, luego tú mismo dejas aparte la letra que le hayas puesto, cantas porque te gusta". 
En los tres grupos hubo consenso sobre la conveniencia de utilizar una amplia variedad de estilos musicales en el aula de Primaria como, por ejemplo, el pop, el rock, el reggae o la clásica, entre otros, exceptuando el reggaetón. Todos los participantes consideraron inadecuado su uso. "Reggaetón no, hasta lo prohibiría" (Emilia), "las letras no son muy indicadas. No parece que pueda llegar a potenciar algo" (Fátima). Aunque hubo una persona que señaló que, en ocasiones, y debido a que es un tipo de música de gran uso entre los jóvenes, podrían cambiarse las letras y adecuarlas al contexto en el que se va a utilizar: "con el reggaetón se puede cambiar la letra, lo que les gusta [al alumnado] es el ritmo" (Claudia).

También expresaron que tiene que haber más creatividad a la hora de plantear actividades musicales en las aulas. Sofía opinó que "se pueden utilizar todo tipo de objetos sonoros, además de los instrumentos tradicionales, inclusive los creados por el propio alumnado, de manera que disfruten haciendo música y experimentando con el sonido”.

Por último, se apuntó que la música tiene una presencia escasa, actualmente, en su formación universitaria. Miriam se preguntó: ¿̨por qué el resto de asignaturas están todos los años en la carrera, sumando más de 40 créditos, y la música solo 6? Debería tener más espacio”.

\section{Discusión y conclusiones}

Este estudio exploratorio-descriptivo se centró en profundizar sobre los significados que otorga un grupo de futuros maestros a sus experiencias musicales vitales y en cómo estas podrían incidir en su futura labor docente.

Los estudiantes integraron eventos que tuvieron impacto emocional en sus vidas y en su proceso de aprendizaje por medio de las experiencias narradas en las que la música tenía un papel relevante (Burnard \& Björk, 2010). La reflexión conjunta, mediante los grupos de discusión, propició una forma de expresión colectiva (Monereo et al., 2015) en la que las experiencias compartidas supusieron una oportunidad para el desarrollo personal desde el autoconocimiento (Valdés et al., 2016). Es por ello que los incidentes musicales relatados fueron vinculados por los participantes con la introspección personal, produciéndose una identificación entre música y emociones, sensaciones, recuerdos y estados de ánimo (Burnard, 2005), que fueron en su mayoría positivas y consideradas importantes en sus experiencias vitales (Hebert, 2009).

Los incidentes musicales narrados fueron ubicados por los estudiantes en contextos formales e informales, constituyéndose como espacios que contribuyen a configurar su identidad personal (Baker, 2005, 2014), una identidad construida sobre los itinerarios vitales de cada uno de los estudiantes desde la experiencia musical (Touriñán \& Longueira, 2010). Los contextos educativos formales fueron los más asociados a una falta de experiencias prácticas con la música mientras que los ámbitos no formales e informales se asociaron al disfrute y estaban relacionados con la familia y los amigos. Esto nos lleva a repensar la forma en que se puede incluir la música en el aula, utilizando sus posibilidades en la educación (Touriñán \& Longueira, 2010) y teniendo en cuenta las experiencias que provienen de situaciones externas al ámbito escolar, muchas veces más significativas que las ocurridas dentro de las aulas y fundamentales para la construcción integral de la persona. Es decir, una educación que contemple la interacción persona, sociedad y contexto (Garvis \& Pendergast, 2012).

Como señala Burbules (2012), hoy en día los límites entre los ámbitos formal, no formal e informal se difuminan y la separación que pueda existir entre ellos es más de tipo físico que de verdaderas diferencias en las posibilidades formativas de cada uno; de esta manera, cada persona teje su propio entorno de aprendizaje, en el que existen interacciones entre todos los ámbitos y contextos en que se relaciona. Por otra parte, los contextos informales desempeñan un importante papel a la hora de comprender quiénes queríamos ser y en quiénes nos hemos convertido. Según los resultados obtenidos, las referencias musicales también provienen de situaciones externas al ámbito escolar, como la familia y los amigos. 
Por otro lado, si mediante las experiencias narradas se puso de manifiesto una apertura de los estudiantes en cuanto a que el recuerdo de las emociones vividas facilitó la introspección personal, esto también logró que estos futuros docentes se conectasen con la construcción de la identidad profesional (Philpott, 2016; Gorzoni \& Davis, 2017). Desde esta mirada, todos ellos reflexionaron sobre las posibilidades didácticas de la música y su potencial en el aula (Burnard, 2005). Por un lado, la música ha sido percibida como un valioso y necesario recurso que puede contribuir al desarrollo emocional de las personas (Campayo \& Cabedo, 2016). Algunas de las ideas que surgieron al respecto están relacionadas con la introspección, la identificación y gestión de emociones, la expresión y la creatividad. También se mostró interés por incorporar una variedad de estilos musicales y una apertura mental hacia prácticas sonoras relacionadas con la experimentación y la exploración (Brinkman, 2010). Asimismo, se ha subrayado la importancia otorgada a la música en relación con la interdisciplinariedad y la participación (Young, 2018) sobre todo, enfocada desde la colaboración docente.

Es reseñable que estos futuros docentes incidiesen especialmente en la necesidad de facilitar, desde las primeras etapas escolares, experiencias musicales donde se produzca la conexión entre el mundo musical particular del alumnado y el del aula, en ocasiones percibido como limitado o poco estimulante y no como un espacio vital, donde se entretejen afectos e intersubjetividades (Vargas et al., 2017).

Por último, se ha constatado la preocupación ante la escasa presencia de la educación musical tanto en los programas escolares como en los universitarios, lo cual concuerda con la idea de (Hennessy, 2017) sobre la limitada presencia de la música en la formación inicial de maestros.

La investigación futura indica la necesidad de ofrecer una formación que invite al alumnado a la reflexión, desde la apertura introspectiva y la comprensión de los múltiples significados que la música puede tener. Las autoras de este artículo invitamos a otros docentes a realizar experiencias en esta línea que avancen hacia un desarrollo curricular en el ámbito de la formación inicial del profesorado, donde la música esté más presente.

\section{Referencias}

Adler, A. (2012). Rediscovering musical identity through narrative in pre-service teacher education.

In M.S. Barret, \& S. L. Stauffer (Ed.), Narrative sundings: An anthology of narrative inquiry in music education (pp. 161-178). Springer.

Andréu, J. (2001). Técnicas de análisis de contenido: Una revisión actualizada. Fundación Centro de Estudios Andaluces. http://mastor.cl/blog/wp-content/uploads/2018/02/Andreu.-analisis-de-contenido.-34pags-pdf.pdf

Angrosino, M. (2012). Etnografía y observación participante en investigación cualitativa. Morata.

Baker, D. (2005). Music service teachers' life histories in the United Kingdom with implications for practice. International Journal of Music Education, 23(3), 263-277. https://doi. org/10.1177/0255761405058243

Baker, D. (2014). Visually impaired musicians' insights: Narratives of childhood, lifelong learning and musical participation. British Journal of Music Education, 31(2), 113-135. https://doi.org/10.1017/ S0265051714000072

Beineke, V. (2012). A reflexão sobre a prática na pesquisa e formação do professor de música. Cadernos de Pesquisa, 42(145), 180-202. http://publicacoes.fcc.org.br/index.php/cp/article/view/53/69

Bilbao, G., \& Monereo, C. (2011). Identificación de incidentes críticos en maestros en ejercicio: Propuestas para la formación permanente. Revista Electrónica de Investigación Educativa, 13(1), 135-151. http://redie.uabc.mx/vol13nol/contenido-bilbaomonereo.html 
Bolívar, A., \& Domingo, J. (2006). La investigación biográfica y narrativa en Iberoamérica: Campos de desarrollo y estado actual. Forum: Qualitative Social Research, 7(4). http://www.qualitative-research. net/fqs-texte/4-06/06-4-12-s.htm.

Bolívar, A., Domingo, J., \& Fernández, M. (2001). La investigación biográfico-narrativa en educación: Enfoque $y$ metodologia. La Muralla.

Brinkman, D. J. (2010). Teaching creatively and teaching for creativity. Arts Education Policy Review, 111(2), 48-50. https://doi.org/10.1080/10632910903455785

Burbules, N. C. (2012). El aprendizaje ubicuo y el futuro de la enseñanza. Encounters/Encuentros/Rencontres on Education, 13, 3-14. https://dialnet.unirioja.es/servlet/articulo?codigo $=4100463$

Burnard, P. (2005). El uso del mapa de incidentes críticos y la narración para reflexionar sobre el aprendizaje musical. Revista Electrónica Complutense de Investigación en Educación Musical, 2(2). http://revistas. ucm.es/index.php/RECI/article/view/RECI0505110002A

Burnard, P., \& Björk, C. (2010). Using student voice research to understand and improve musical learning. In J. Finney \& Ch. Harrison (Ed.), Whose music education is it? The role of the student voice (pp. 24-32). National Association of Music Educators NAME.

Callejo, J. (2001). El grupo de discusión: Introducción a una práctica de investigación. Ariel Practicum.

Campayo, E., \& Cabedo, A. (2016). Música y competencias emocionales: Posibles implicaciones para la mejora de la educación musical. Revista Electrónica Complutense de Investigación en Educación Musical, 13, 124-139. https://doi.org/10.5209/RECIEM.51864

Carrillo, C., \& Vilar, M. (2016). Percepciones del profesorado de música sobre competencias profesionales necesarias para la práctica. Opción, 32(n. especial 7), 358-382. https:/dialnet.unirioja.es/ ejemplar/457279

Castañeda, M.A. (2013). Identidades en proceso de formación. Cuadernos de Pedagogía, (436), 14-17. http://www.cuadernosdepedagogia.com/content/Inicio.aspx

Ceballos, N., \& Susinos, T. (2014). La participación del alumnado en los procesos de formación y mejora docente. Una mirada a través de los discursos de orientadores y asesores de formación. Profesorado. Revista de Curriculum y Formación del Profesorado, 18(2), 228-244. https://www.ugr.es/ recfpro/ rev182COL5.pdf

Colomo, E., \& Domínguez, R. (2015). Definiendo identidades: El "canciograma” como herramienta metodológica de autoconocimiento. Revista Electrónica Iberoamericana sobre Calidad, Eficacia y Cambio en Educación, 13(2), 131-146. https://revistas.uam.es/reice/article/view/2794

Cornejo, M., Mendoza, F., \& Rojas, R. C. (2008). La investigación con relatos de vida: Pistas y opciones del diseño metodológico. PSYKHE, 17(1), 29-39. http://dx.doi.org/10.4067/S071822282008000100004

Denzin, N. K., \& Lincoln, Y. S. (2012). Manual de investigación cualitativa. Gedisa.

Farías, L., \& Montero, M. (2005). De la transcripción y otros aspectos artesanales de la investigación cualitativa. International Journal of Qualitative Methods, 4(1), 1-14. https://journals.sagepub.com/ doi/pdf/10.1177/160940690500400104

Fernández-Jiménez, A., \& Jorquera-Jaramillo, M.-C. (2017). El sentido de la educación musical en una educación concebida como motor de la economía del conocimiento: Una propuesta de marco filosófico. Revista Electrónica Complutense de Investigación en Educación Musical, 14, 95-107. https://doi.org/10.5209/RECIEM.54834

García, E., \& Lorente, R. (2017). De receptor pasivo a protagonista activo del proceso de enseñanzaaprendizaje: Redefinición del rol del alumnado en la Educación Superior. Opción, 33(84), 120-153. https://dialnet.unirioja.es/servlet/articulo? codigo $=6402368$

Garvis, S., \& Pendergast, D. (2012). Storying music and the arts education: The generalist teacher voice. British Journal of Music Education, 29(1), 107-123. https://doi.org/10.1017/S0265051711000386 
Gorzoni, S., \& Davis, C. (2017). O conceito de profissionalidade docente nos estudos mais recentes. Cadernos de Pesquisa, 47(166), 1396-1413. https://doi.org/10.1590/198053144311

Hebert, D.G. (2009). Musicianship, musical identity, and meaning as embodied practice. In Th. A. Regelski, \& J. T. Gates (Eds.), Music Education for Changing Times (pp. 39-55). Springer.

Hennessy, S. (2017). Approaches to increasing the competence and confidence of student teachers to teach music in primary schools. Education 3-13, 45(6), 689-700. https://doi.org/10.1080/03004279.2017.1347130

Lorenzo, O. (2011). Análisis cualitativo de textos sobre multi e interculturalidad. DEDiCA. Revista de Educação e Humanidades, (1), 535-546. https://doi.org/10.30827/dreh.v0i1.7186

Luque, P. J., \& Cruz, C. M. (2016). Prácticas cualitativas para la investigación sobre las artes. In M. I. Moreno, \& M. P. López-Peláez (Coords.), Reflexiones sobre investigación artística e investigación educativa basada en las artes (pp. 43-59). Síntesis.

Monereo, C., \& Badía, A. (2011). Los heterónimos del docente: Identidad, selfs y enseñanza. In C. Monereo, \& J. I. Pozo (Eds.), La identidad en psicología de la educación: Enfoques actuales, utilidad $y$ limites (pp. 59-77). Narcea.

Monereo, C., Monte, M., \& Andreucci, P. (2015). La gestión de incidentes criticos en la universidad. Narcea.

Philpott, C. (2016). Narratives as a vehicle for mentor and tutor knowledge during feedback in initial teacher education. Teacher Development, 20(1), 57-75. http://www.tandfonline.com/doi/ full/10.1080/13664530.2015.1108927? src=recsys

Pitts, S. (2012). Chances and choices: Exploring the impact of music education. Oxford University Press.

Regelski, Th. A., \& Gates, J. T. (2009). Music education for changing times. Springer.

Riaño, M.E. (2017). Creación sonora a partir de las narrativas biográficas en el aula de música: narrativas sonoras. In A. Murillo, \& M. Díaz (Coords.), La mecánica de la creación sonora (pp. 109-134). Institut de Creativitat i Innovacions Educatives de la Universitat de València.

Richardson, C. (2012). Narratives from Preservice Music Teachers: Hearing their voices while singing with the Choir. In M. S. Barret, \& S. L. Stauffer (Eds.), Narrative soundings: An anthology of narrative inquiry in music education (pp. 179-200). Springer.

Sánchez-Sánchez, G. I., \& Jara-Amigo, X. E. (2015). Visión del trabajo docente en el ámbito de la evaluación, que comienza a construir el profesorado en formación, a partir del uso de incidentes críticos en los procesos de formación práctica. Revista Electrónica Educare, 19(2), 231-255. doi: http://dx.doi. org $/ 10.15359 /$ ree.19-2.14

Silvennoinen, M. (2001). Relatos sobre deporte e identidad en mujeres y hombres. In J. Devís (Ed.), La educación física, el deporte y la salud en el siglo XXI (pp. 203-212). Marfil.

Sockman, B. R., \& Sharma, P. (2008). Struggling toward a transformative model of instruction: It's not so easy!. Teaching and Teacher Education, 24(4), 1070-1082. https://doi.org/10.1016/j.tate.2007.11.008

Sparkes, A. C., \& Devís, J. (2007). Investigación narrativa y sus formas de análisis: Una visión desde la educación física y el deporte. In W. Moreno, \& S. Maryory (Eds.), Educación, cuerpo y ciudad. El cuerpo en las interacciones e instituciones sociales (pp. 43-68). Funámbulos Editores.

Touriñán, J. M., \& Longueira, S. (2010). La música como ámbito de educación. Educación 'por' la música y educación 'para' la música. Teoría de la Educación, 22(2), 151-181. https://doi.org/10.14201/8300

Tripp, D. (2012). Critical incidents in teaching: Developing professional judgement. Routledge.

Valdés, A., Coll, C., \& Falsafi, L. (2016). Experiencias transformadoras que nos confieren identidad como aprendices: Las experiencias clave de aprendizaje. Perfiles Educativos, 38(153), 168-184. https://doi. org/10.22201/iisue.24486167e.2016.153.57643

Vargas, C., Ledezma Delgado, L. J., \& Castro, S. (2017). El Aula, espacio de sentido y significado [Tesis de Maestría, Universidad de Manizales]. RiDUM - Repositório Institucional Universidad de Manizales. https://ridum.umanizales.edu.co/xmlui/bitstream/handle/20.500.12746/3314/ Sandra\%20Milena\%20Castro\%20Plazas\%2c\%202017.pdf?sequence=2\&isAllowed=y 
Verd, J. M., \& Lozares, C. (2016). Introducción a la investigación cualitativa: Fases, métodos y técnicas. Síntesis. Young, G. (2018). Creative interdisciplinary in the arts. In N. H. Hensel (Ed.), Exploring, experiencing, and envisioning integration in US Arts Education (pp. 15-26). Palgrave MacMillan.

\section{Nota sobre la autoría}

Este artículo fue construido colectivamente por las autoras.

\section{Disponibilidad de datos}

Los datos subyacentes al texto de la investigación se informan en el artículo.

\section{Cómo citar este artículo}

De Alba Eguiluz, B., Arriaga-Sanz, C., \& Riaño-Galán, M. E. (2021). Experiencias musicales a través del relato autobiográfico en la formación docente. Cadernos de Pesquisa, 51, Artículo e07388. https://doi.org/10.1590/198053147388 